\title{
Quantitative Muscle Ultrasonography in Carpal Tunnel Syndrome
}

\author{
Hyewon Lee, MD, Sungju Jee, MD, PhD, Soo Ho Park, MD, \\ Seung-Chan Ahn, MD, Juneho Im, MD, Min Kyun Sohn, MD, PhD
}

Department of Rehabilitation Medicine, Chungnam National University School of Medicine, Daejeon, Korea

\begin{abstract}
Objective To assess the reliability of quantitative muscle ultrasonography (US) in healthy subjects and to evaluate the correlation between quantitative muscle US findings and electrodiagnostic study results in patients with carpal tunnel syndrome (CTS). The clinical significance of quantitative muscle US in CTS was also assessed.

Methods Twenty patients with CTS and 20 age-matched healthy volunteers were recruited. All control and CTS subjects underwent a bilateral median and ulnar nerve conduction study (NCS) and quantitative muscle US. Transverse US images of the abductor pollicis brevis (APB) and abductor digiti minimi (ADM) were obtained to measure muscle cross-sectional area (CSA), thickness, and echo intensity (EI). EI was determined using computerassisted, grayscale analysis. Inter-rater and intra-rater reliability for quantitative muscle US in control subjects, and differences in muscle thickness, CSA, and EI between the CTS patient and control groups were analyzed. Relationships between quantitative US parameters and electrodiagnostic study results were evaluated.

Results Quantitative muscle US had high inter-rater and intra-rater reliability in the control group. Muscle thickness and CSA were significantly decreased, and EI was significantly increased in the APB of the CTS group (all $\mathrm{p}<0.05)$. EI demonstrated a significant positive correlation with latency of the median motor and sensory NCS in CTS patients $(\mathrm{p}<0.05)$.

Conclusion These findings suggest that quantitative muscle US parameters may be useful for detecting muscle changes in CTS. Further study involving patients with other neuromuscular diseases is needed to evaluate peripheral muscle change using quantitative muscle US.
\end{abstract}

Keywords Carpal tunnel syndrome, Quantitative muscle ultrasonography, Electrodiagnostic study, Echo intensity, Peripheral neuropathy

Received March 28, 2016; Accepted May 26, 2016

Corresponding author: Min Kyun Sohn

Department of Rehabilitation Medicine, Regional Rehabilitation Center, Chungnam National University Hospital, 282 Munhwa-ro, Jung-gu, Daejeon 35015, Korea. Tel: +82-42-338-2460, Fax: +82-42-338-2461, E-mail: mksohn@cnuh.co.kr

ORCID: Hyewon Lee (http://orcid.org/0000-0003-3105-4071); Sungju Jee (http://orcid.org/0000-0002-9400-9609); Soo Ho Park (http://orcid.org/00000002-1815-5196); Seung-Chan Ahn (http://orcid.org/0000-0003-2295-6233); Juneho Im (http://orcid.org/0000-0002-2296-7832); Min Kyun Sohn (http://orcid.org/0000-0002-2548-545X).

(a) This is an open-access article distributed under the terms of the Creative Commons Attribution Non-Commercial License (http://creativecommons.org/ licenses/by-nc/4.0) which permits unrestricted noncommercial use, distribution, and reproduction in any medium, provided the original work is properly cited. Copyright $\odot 2016$ by Korean Academy of Rehabilitation Medicine 


\section{INTRODUCTION}

Denervation muscle results in significant weakness and muscle atrophy. Early and accurate detection of muscle denervation is important to prevent muscle weakness. Electrodiagnostic study is the gold standard for evaluating muscle denervation in peripheral neuropathy $[1,2]$. However, due to pain caused by needle insertion, an electrodiagnostic study can be invasive and uncomfortable for patients. In addition, patient cooperation is required for obtaining accurate results during this test $[3,4]$. Interest in high-resolution ultrasonography (US) is increasing $[5,6]$. Compared to an electrodiagnostic study, musculoskeletal US is relatively noninvasive and more readily available. US can also provide real-time and dynamic information on patients [7].

Several studies have demonstrated that neuromuscular diseases can causes changes in the normal ultrasound image of the muscle $[8,9]$ and echo intensity (EI) $[10,11]$. Normal muscle tissue is echolucent, and ultrasound is transmitted through the muscle tissue easily but it is reflected from the fibrous tissue. In imaging studies, fat and collagen replacement and muscle atrophy make muscle tissue heterogeneous. Therefore, muscle EI increases with fatty infiltration and fibrous changes [12,13]. High muscle EI has been suggested as evidence of muscle changes due to neuropathy and myopathy [14-16].

In neuromuscular disorders, decreased muscle thickness is apparent. Muscle US could be used to detect muscle changes in the early phase of amyotrophic lateral sclerosis. Quantitative analysis demonstrated a significant increase in EI in all muscles and a bilateral decrease in muscle thickness of the biceps brachii, forearm flexors, and quadriceps femoris [13,17].

Carpal tunnel syndrome (CTS) is a common disorder resulting from compression of the median nerve at the wrist [18]. Diagnosis of CTS is based on clinical signs and symptoms and it is confirmed by electrodiagnostic studies. Quantitative US of the median nerve-innervated muscles can be used to evaluate CTS. However, there are few reports on the quantitative and objective assessment of fatty infiltration of muscle in focal neuropathy using US. In a study by Kim et al. [19], the ratio of thenar muscle EI (mean pixel brightness) to hypothenar muscle EI and the muscle inhomogeneity ratio (pixel standard deviation) were significantly higher in the patient group than in the control group, demonstrating that the EI ratio and inhomogeneity ratio were useful variables to evaluate disease severity and the presence of muscle denervation in patients with CTS.

In this study, we assessed the reliability of quantitative muscle US in healthy control subjects, differences in US findings between the control and CTS groups, and the correlations between quantitative muscle US findings and electrodiagnostic study results in patients with CTS. We also evaluated the clinical significance of quantitative muscle US in CTS.

\section{MATERIALS AND METHODS}

\section{Subjects}

Twenty patients who were confirmed to have CTS by electrodiagnostic study and who agreed to participate in the study were recruited. To establish a clear definition of CTS, electrodiagnostic criteria were used for inclusion. Patients with medical conditions that could cause peripheral neuropathy such as diabetes mellitus, malignancy, or other endocrine diseases were excluded. Patients with a previous history of wrist fracture or other injury of the upper extremities, neuromuscular junction disorder, motor neuron disease, central nervous system disorder, or peripheral nerve disease other than CTS were also excluded. To determine the reliability of quantitative US, 20 age-matched volunteers with no history of neuromuscular disease were recruited.

The study was approved by Chungnam National University Hospital Institutional Review Board (CNUH 201112) and all participants understood the purpose of the study.

\section{Methods \\ Ultrasonography}

Transverse US images of the median-innervated abductor pollicis brevis (APB) muscle and the ulnar-innervated abductor digiti minimi (ADM) muscle were obtained to measure muscle cross-sectional area (CSA), thickness, and EI. US was performed with the subject sitting in a comfortable position with the upper arm in line with the trunk, the elbow flexed to $90^{\circ}$, the forearm supinated, the wrist in neutral position, and the thumb interphalangeal joint fully extended. During the measurements, subjects were asked to fully relax. For APB measurement, the 
probe was placed parallel to the ground at the midpoint of the first metacarpal bone, perpendicular to the long axis of the bone. ADM measurement was performed on the ulnar side of the palm, at the half of the fifth metacarpal bone, perpendicular to the long axis of the fifth metacarpal bone (Fig. 1).

Quantitative muscle US was conducted using an ultrasound machine with a 3-12 MHz linear transducer (EnVisor; Philips, Cleveland, OH, USA). The muscle CSA and EI were measured using B-mode US under the same equipment settings including gain $50 \mathrm{~dB}$ and depth of 3 $\mathrm{cm}$. A visible ultrasound gel layer with a thickness of 2-7 $\mathrm{mm}$ was maintained between the transducer and the skin on the ultrasound monitor for objective and consistent evaluation of the region of interest (ROI). The presence of a gel layer resulted in minimal compression from the transducer to the skin and the underlying tissues. Thickness of the APB and ADM muscles was measured between the upper and lower margins of the muscles at the thickest part. To measure the CSA, transverse images of the muscles were obtained at the level of maximum CSA. The CSAs of the APB and ADM muscles were determined by outlining the muscle contour by the internal rim using the area measurement software of the US system. Both arms were measured (Fig. 2).

EI was determined using computer-assisted, grayscale analysis. ROIs were drawn for analysis of muscle EI using the line along the muscle fascia. Grayscale images were used to quantify muscle EI within each ROI. A grayscale image consists of achromatic color pixels with different brightness values ranging from 0 (pure black) to 255 (pure white), resolution $800 \times 600$ pixels, 8 bit [20]. We analyzed muscle EI by using the standard histogram function of a graphics package (Adobe Photoshop CS5; Adobe Systems Inc., San Jose, CA, USA) (Fig. 3).

To assess the inter-rater reliability of quantitative US, two blind examiners conducted ultrasound examinations in each control subject on the same day. Examinations were performed by one experienced examiner and one novice examiner. To assess intra-rater reliability, one examiner measured each muscle 3 times.

\section{Electrodiagnostic study}

All control and CTS subjects underwent a bilateral median and ulnar nerve conduction study (NCS) using the Nicolet Viking IV Electrodiagnostic System (Nicolet Instrument Inc., Madison, WI, USA). Electrodiagnostic study was performed with skin temperature maintained above $34^{\circ} \mathrm{C}$. The median motor NCS was performed with stimulation at the wrist and the antecubital fossa and recording from the APB. The ulnar motor NCS was performed with stimulation at the wrist and the elbow and
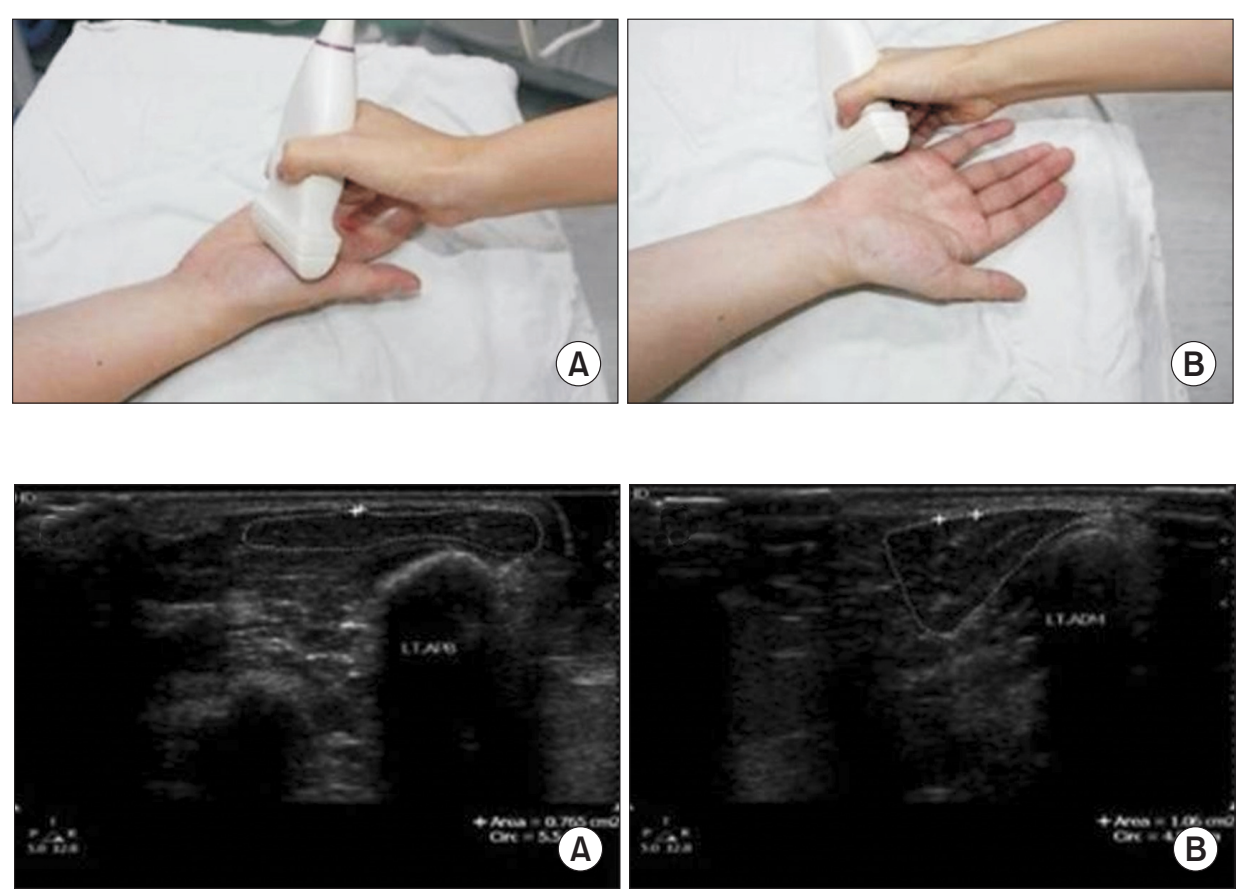

Fig. 2. Transverse ultrasonography images of the left abductor pollicis brevis (APB) and abductor digiti minimi (ADM) of a control subject. Calculation of muscle thickness and cross-sectional area in the APB (A) and ADM (B). 


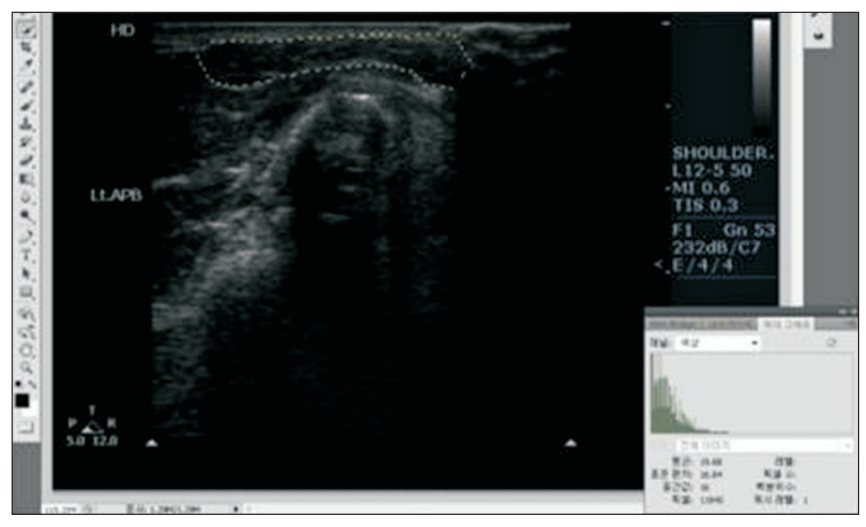

Fig. 3. Echo intensity (EI) analysis of ultrasound images. Grayscale images were used to determine muscle EI within each region of interest (ROI). The mean and standard deviation of the pixel brightness in each ROI were automatically calculated by Photoshop software.

recording from the ADM. The onset latency, amplitude of the compound muscle action potential (CMAP), and conduction velocity of each nerve were recorded. The antidromic median and ulnar sensory nerve action potential (SNAP) responses over the second and fifth finger were recorded. Stimulation of the median and ulnar sensory nerves at the wrist level was performed $14 \mathrm{~cm}$ proximal to the recording electrodes. Additionally, stimulation of the median sensory nerve at the palm level was performed $7 \mathrm{~cm}$ proximal to the recording electrodes. The onset latency and amplitude of the SNAP were recorded.

CTS was diagnosed based on the following criteria: (1) median sensory nerve conduction study, distal latency $>3.5$ ms; (2) median motor nerve conduction study, distal latency $>4.3 \mathrm{~ms}$; (3) difference between distal sensory latency of the median nerve at the wrist and palm level $>1.6 \mathrm{~ms}$ [21]. Based on the electrodiagnostic findings, patients' hands were divided into 5 subgroups using the severity grading system based on the modified Padua criteria [22].

\section{Statistical analysis}

Intraclass correlation coefficient (ICC) was used to quantify inter-rater and intra-rater reliability for quantitative US in control subjects. We interpreted the results considering that an ICC above 0.75 indicated excellent reliability, ICC between 0.4 to 0.75 indicated fair to good reliability, and ICC below 0.4 had poor reliability [23].

Differences in muscle thickness, CSA, and EI between the CTS patient and control groups were analyzed using
Table 1. Demographic characteristics

\begin{tabular}{|c|c|c|}
\hline Characteristic & CTS group & Control group \\
\hline \multicolumn{3}{|l|}{ Sex } \\
\hline Male & 10 & 10 \\
\hline Female & 10 & 10 \\
\hline \multicolumn{3}{|l|}{ Lesion side } \\
\hline Bilateral & 16 & \\
\hline Right & 2 & \\
\hline Left & 2 & \\
\hline Age (yr) & $54.9 \pm 11.5$ & $52.7 \pm 14.9$ \\
\hline Height $(\mathrm{cm})$ & $164.3 \pm 9.9$ & $163.7 \pm 10.1$ \\
\hline Weight (kg) & $65.9 \pm 12.5$ & $63.4 \pm 13.9$ \\
\hline \multicolumn{3}{|l|}{ Hand dominance } \\
\hline Right & 20 & 18 \\
\hline Left & 0 & 2 \\
\hline \multicolumn{3}{|c|}{ CTS severity (hands) ${ }^{a}$} \\
\hline Minimal & 2 & - \\
\hline Mild & 26 & - \\
\hline Moderate & 8 & - \\
\hline Duration (mo) ${ }^{\mathrm{b})}$ & $9.8 \pm 13.2$ & - \\
\hline
\end{tabular}

Values are presented as number or mean \pm standard deviation.

CTS, carpal tunnel syndrome.

${ }^{a}$ U) Using the severity grading system based on the modified Padua criteria. ${ }^{\mathrm{b})}$ Duration after CTS symptoms.

the independent $\mathrm{t}$-test. The $\mathrm{p}$-values $<0.05$ were considered statistically significant. Pearson correlation coefficient and corresponding $\mathrm{p}$-values were used to evaluate the relationships between quantitative US parameters and electrodiagnostic study results. All analyses were performed using SPSS Statistics ver. 19.0 (SPSS Inc., Chicago, IL, USA). All measured values are reported as mean \pm standard deviation.

\section{RESULTS}

\section{Subject characteristics}

A total of 20 patients with CTS (bilateral, 16; right, 2; left, 2; mean age, $54.9 \pm 11.5$ years) and 20 control subjects (mean age, $52.7 \pm 14.9$ years) were included in this study. Baseline characteristics of all subjects are shown in Table 1. There were no differences in sex, age, height, and weight between the two groups. 
Table 2. Inter-rater and intra-rater reliability of ultrasonography parameters in control subjects

\begin{tabular}{|c|c|c|c|c|}
\hline & \multicolumn{2}{|c|}{ APB } & \multicolumn{2}{|c|}{ ADM } \\
\hline & ICC & p-value & ICC & p-value \\
\hline \multicolumn{5}{|c|}{ Inter-rater reliability } \\
\hline Thickness & 0.537 & 0.051 & 0.853 & 0.00 \\
\hline CSA & 0.820 & 0.00 & 0.968 & 0.00 \\
\hline Echo intensity & 0.897 & 0.02 & 0.837 & 0.001 \\
\hline \multicolumn{5}{|c|}{ Intra-rater reliability } \\
\hline Thickness & 0.857 & 0.02 & 0.858 & 0.00 \\
\hline CSA & 0.939 & 0.001 & 0.940 & 0.00 \\
\hline Echo intensity & 0.949 & 0.00 & 0.773 & 0.00 \\
\hline
\end{tabular}

APB, abductor pollicis brevis; ADM, abductor digiti minimi; ICC, intraclass correlation coefficient; CSA, cross-sectional area.

Table 3. Values of muscle ultrasonography in the CTS and control groups

\begin{tabular}{lrrr}
\hline & CTS group & Control group & p-value \\
\hline APB & & & \\
Thickness $(\mathrm{cm})$ & $0.43 \pm 0.08$ & $0.59 \pm 0.15$ & $0.00^{*}$ \\
CSA $\left(\mathrm{mm}^{2}\right)$ & $1.12 \pm 0.28$ & $1.59 \pm 0.22$ & $0.00^{*}$ \\
Echo intensity & $28.13 \pm 5.15$ & $19.93 \pm 2.05$ & $0.00^{*}$ \\
ADM & & & \\
Thickness (cm) & $1.03 \pm 0.25$ & $0.95 \pm 0.20$ & 0.53 \\
CSA $\left(\mathrm{mm}^{2}\right)$ & $1.47 \pm 0.43$ & $1.13 \pm 0.28$ & 0.10 \\
\hline Echo intensity & $23.69 \pm 6.53$ & $24.07 \pm 2.05$ & 0.72 \\
\hline
\end{tabular}

Values are presented as mean \pm standard deviation. CTS, carpal tunnel syndrome; APB, abductor pollicis brevis; ADM, abductor digiti minimi; CSA, cross-sectional area.

${ }^{*} \mathrm{p}<0.05$ by independent $\mathrm{t}$-test.

Quantitative muscle ultrasonography in control subjects

The results of the inter-rater and intra-rater reliability analyses in the control group are shown in Table 2 . The quantitative US findings demonstrated excellent inter-rater reliability for CSA and EI of the APB; and for thickness, CSA, and EI of the ADM based on ICC values above 0.75. However, measurement of APB thickness showed fair to good inter-rater reliability between a novice assessor and an experienced assessor. Using 3 trials per rater, excellent intra-rater reliability was observed in thickness, CSA, and EI of the APB (ICC $=0.857-0.949$ ) and ADM (ICC $=0.773-$ 0.940). Additionally, quantitative muscle US findings did not significantly differ between men and women or the left hand and the right hand in the control group.

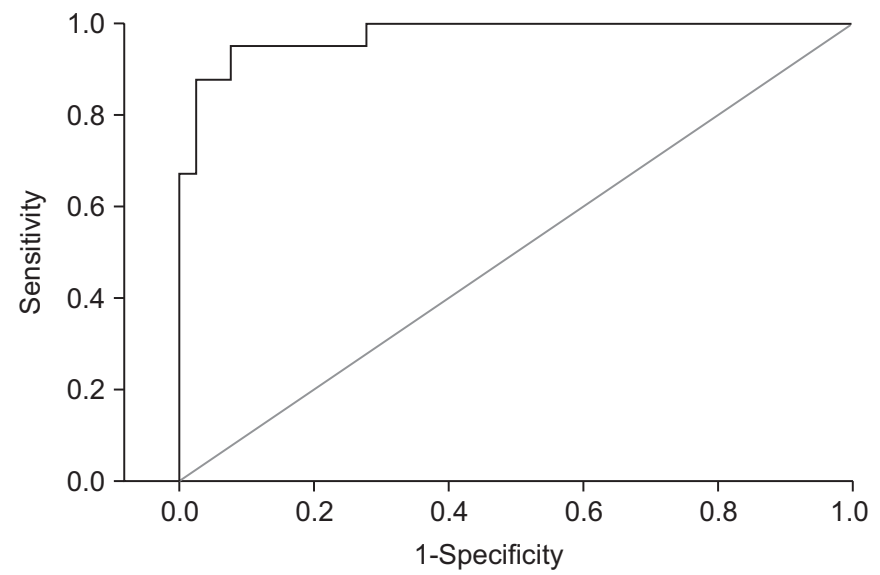

Fig. 4. Receiver operating characteristic (ROC) curve for abductor pollicis brevis muscle echo intensity to distinguish patients with carpal tunnel syndrome from control subjects. The optimal cutoff point was 22.60 (sensitivity $92.5 \%$, specificity $92.5 \%)$.

\section{Comparison of ultrasonographic parameters between} the CTS and control groups

Comparison of quantitative muscle US parameters between CTS patients and control subjects are shown in Table 3. The muscle thickness and CSA of the APB were significantly decreased in CTS patients $(\mathrm{p}<0.05)$, and EI of the APB was significantly increased in CTS patients $(\mathrm{p}<0.05)$. However, results of ADM measurements did not differ significantly between controls and patients.

To determine the optimal cutoff point for EI to discriminate the CTS patient group from the control group, receiver operating characteristic (ROC) curve analysis was performed (Fig. 4). The optimal cutoff point of EI to define CTS was 22.60 (sensitivity $92.5 \%$, specificity $92.5 \%$ ). 
EI had a relatively high area under the curve (AUC=0.976).

Relationship between electrodiagnostic study and quantitative muscle ultrasonography

The correlation between quantitative US and electrodiagnostic parameters in the APB is shown in Table 4. There was no significant correlation between quantitative US and electrodiagnostic parameters in the control group. However, in CTS patients, EI demonstrated a significant positive correlation with the latency of the median motor and sensory NCS $(p<0.05)$. EI was negatively correlated with muscle thickness and CSA in CTS patients $(\mathrm{p}<0.05)$. These significant relationships are shown graphically by using scatter plots (Fig. 5).

\section{DISCUSSION}

The aim of this study was to investigate the differences in quantitative US findings between control subjects and CTS patients and to assess the correlation between electrodiagnostic study results and quantitative muscle US findings.

Musculoskeletal ultrasonography has been established as a diagnostic method for decades. Improvements in technology have led to its broad use in many specializations. One of its main indications is the differentiation of normal and pathological anatomical structures [24]. CTS can be diagnosed by sonography, mainly based on the demonstration of an increase in CSA of the median nerve at the level of the pisiform bone. Additionally, nerve ultrasonography in cases of peripheral neuropathy has the advantage of being able to detect a mass lesion, anatomical variants, and the underlying abnormality [25]. However, there are few reports of CTS diagnosis using quantitative muscle ultrasonography.

On muscle US, the muscle EI was measured in addition to muscle thickness and CSA. Time between sending and receiving the ultrasound pulse determines the location of the corresponding pixel, and the amplitude of the sound wave corresponds to the brightness of the image. The amount of returning echoes per square unit area determines the gray value of the image and that gray value is EI [26]. Muscle EI was determined as mean pixel brightness, and the standard deviation of pixel brightness indicates muscle inhomogeneity $[12,19]$. Changes in muscle echogenicity reflected denervation edema after nerve injury, followed by accumulation of fat and fibrous tissue [27]. In neuropathy or myopathy, muscle EI changes differently. Neurogenic disorders usually lead to an inhomogeneous increase in EI with atrophy. Myopathies generally result in a homogeneous increase in muscle EI, often accompanied by a preserved muscle bulk [26].

In this study, we assessed the reliability of quantitative US of the APB and ADM in control subjects. Excellent inter-rater and intra-rater reliability were observed for EI and CSA measurements in both APB and ADM. The APB thickness showed fair to good inter-rater reliability. Some studies have reported the inter-rater reliability of muscle thickness or CSA. Wallwork et al. [28] studied the intrarater and inter-rater reliability of multifidus muscle thick-

Table 4. Correlation coefficients between quantitative muscle ultrasonography and electrodiagnostic study in the APB of control subjects and CTS patients

\begin{tabular}{|c|c|c|c|c|c|c|c|c|c|}
\hline & \multicolumn{3}{|c|}{ Motor NCS } & \multicolumn{3}{|c|}{ Sensory NCS } & \multirow{2}{*}{ Thickness } & \multirow{2}{*}{ CSA } & \multirow{2}{*}{ EI } \\
\hline & Amp & Lat & CV & Amp & Lat & $\mathrm{CV}$ & & & \\
\hline \multicolumn{10}{|c|}{ Control group } \\
\hline Thickness & 0.29 & -0.12 & -0.03 & -0.12 & -0.39 & 0.01 & 1.00 & - & - \\
\hline CSA & 0.04 & 0.26 & -0.27 & -0.10 & 0.19 & 0.10 & -0.05 & 1.00 & - \\
\hline EI & 0.01 & 0.00 & 0.24 & 0.05 & -0.02 & 0.06 & -0.03 & 0.30 & 1.00 \\
\hline \multicolumn{10}{|l|}{ CTS group } \\
\hline Thickness & 0.18 & -0.17 & 0.10 & 0.10 & -0.17 & 0.23 & 1.00 & - & - \\
\hline CSA & 0.13 & -0.10 & 0.04 & 0.04 & -0.12 & 0.20 & $0.51^{* *}$ & 1.00 & - \\
\hline EI & -0.21 & $0.65^{*}$ & -0.37 & -0.00 & $0.67^{*}$ & -0.65 & $-0.40^{*}$ & $-0.41^{* *}$ & 1.00 \\
\hline
\end{tabular}

APB, abductor pollicis brevis; CTS, carpal tunnel syndrome; NCS, nerve conduction study; Amp, amplitude; Lat, onset latency; CV, conduction velocity; CSA, cross-sectional area; EI, echo intensity.

${ }^{*} \mathrm{p}<0.05,{ }^{* *} \mathrm{p}<0.01$ by Pearson correlation coefficient analysis. 

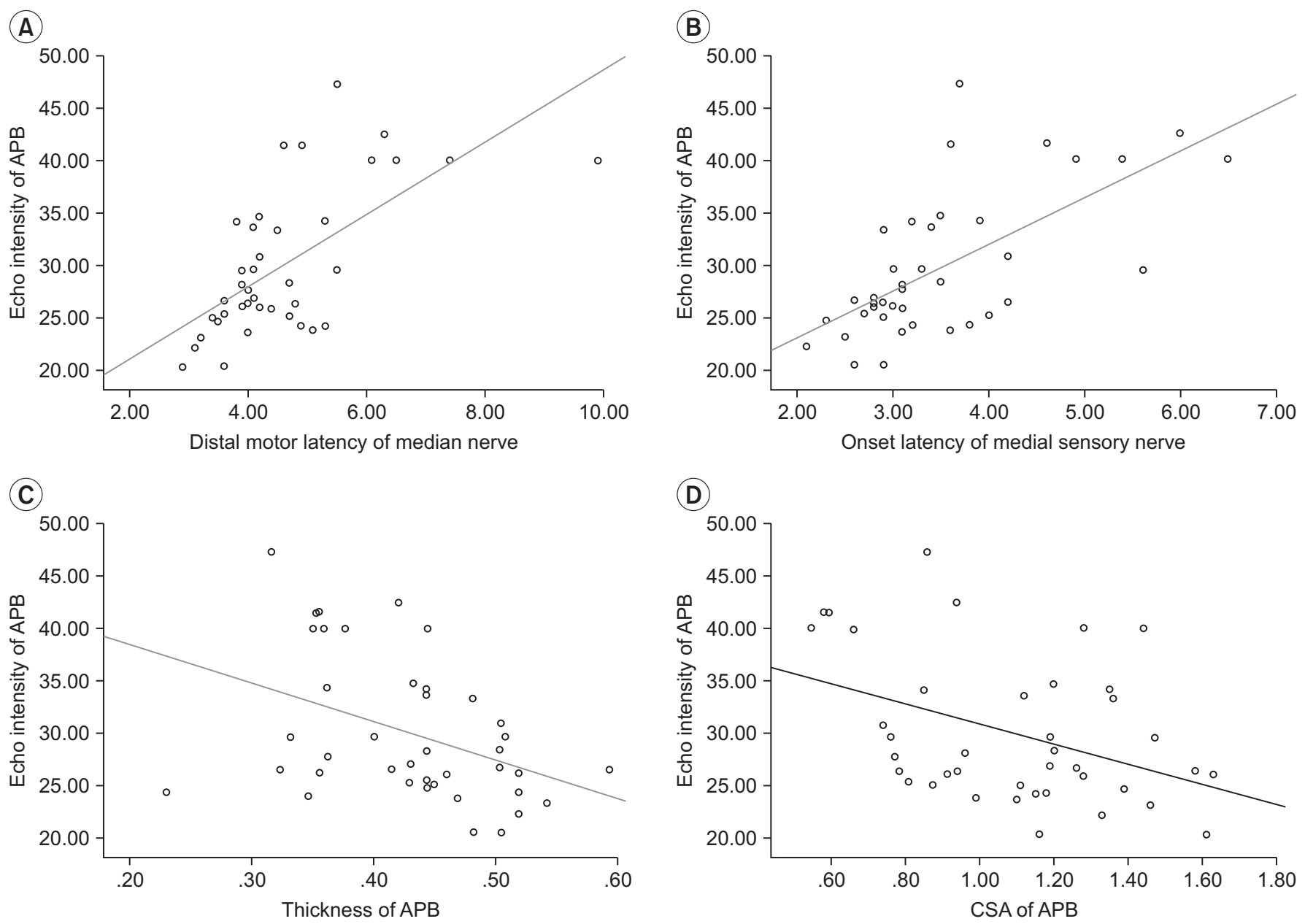

Fig. 5. Pearson correlation scatter plots for study results of echo intensity of abductor pollicis brevis (APB). (A) Distal motor latency of the median nerve $(r=0.65)$. (B) Onset latency of the median sensory nerve $(r=0.67)$. (C) Thickness of APB ( $r=-0.4)$. (D) Cross-sectional area (CSA) of APB ( $r=-0.41)$.

ness measurements in the parasagittal plane, conducted by an experienced assessor and a novice assessor. When a standardized protocol was followed, high inter-rater reliability and intra-rater reliability were demonstrated (ICC $=0.87-0.97$ ). In our study, we found that the ICC for APB thickness was lower than the ICC for CSA and EI. Muscle images in the thenar area are not from a single muscle, i.e., the APB. The APB muscle is flat in shape and close to other small muscles. Therefore, it is difficult to distinguish the muscle boundaries clearly. We further tried to minimize the effect of probe compression by maintaining an ultrasound gel layer with a thickness of 2-7 mm between the transducer and the skin. However, compression of the probe could have occurred and might have affected the muscle thickness.

Arts et al. [13] demonstrated that thickness and EI in the muscles of the upper extremities showed sex differences and a muscle-specific nonlinear correlation with age in volunteers aged 17-90 years. In our study, the impact of age on EI was not assessed because the age range of subjects (range, 43-73 years) was too limited to allow a robust statistical analysis. However, quantitative muscle US findings did not differ according to sex or between the left hand and the right hand in our study. Further study is needed to evaluate the effect of subject characteristics on EI.

In a study by Kim et al. [19], the hands of CTS patients demonstrated a higher EI than those of control subjects. Subgroup analysis demonstrated that the EI measurements in the severe CTS group were significantly higher than those in the moderate CTS group. These results suggest that muscle EI parameters reflect the severity of CTS, 
and US may be able to objectively and quantitatively assess fatty infiltration of the APB muscle. Our study yielded similar results in that the muscle thickness and CSA of the APB were significantly decreased and EI of the APB was significantly increased in the CTS group. In addition, EI of the APB demonstrated a significant positive correlation with the latency of median motor and sensory NCS $(\mathrm{p}<0.05)$ and a negative correlation with muscle thickness and CSA of the APB in CTS patients. However, CSA and thickness of the APB were not significantly correlated with electrodiagnostic study results. Therefore, our results suggest that quantitative muscle US findings, especially EI, have clinical value in the evaluation of muscle change in CTS.

Simon et al. [28] performed quantitative US for the assessment of neuromuscular disorders involving denervation of hand muscles. Quantitative ultrasound of hand muscles, APB, ADM, and first dorsal interosseous muscles, could distinguish between moderate and severe muscle denervation and healthy hand muscles. Ultrasound parameters were predictive of the severity of denervation identified on electromyography (EMG) studies. Muscle thickness and echogenicity change in denervated muscles correlated with the severity of EMG abnormalities. Muscle thickness decreased with increasing severity of EMG abnormalities, while muscle echogenicity increased with increasing severity of denervation.

In the control group, there was no significant correlation between quantitative US findings and electrodiagnostic parameters. Therefore, the amplitude of CMAP in the APB and ADM was not related to the thickness and CSA of APB and ADM in healthy volunteers.

Our study had several limitations. First, the number of enrolled subjects was small. Additionally, this study included only mild to moderate CTS patients. Therefore, it is difficult to generalize these study results to all CTS patients. Further studies with larger sample sizes and different subject populations are needed to increase our understanding of the clinical applicability of this method. Second, we did not evaluate the CSA of median nerves. For the diagnosis of CTS, an increase in CSA of the median nerve is a characteristic feature of ultrasonography and it had been widely studied. Further study is needed to demonstrate the relationship between the CSA of the median nerve and quantitative muscle US findings in CTS.
In conclusion, quantitative muscle US has high interrater and intra-rater reliability in control subjects. Ultrasonographic findings of CTS patients significantly differed from those of the control group. EI of the APB muscle correlated with the electrodiagnostic findings of CTS. Quantitative analysis of muscle thickness, CSA, and EI could be a more objective and reliable method to detect peripheral neuropathy. Therefore, quantitative muscle US may be clinically helpful in determining the presence of CTS. Further study for other neuromuscular diseases is necessary to evaluate peripheral muscles by using quantitative muscle US.

\section{CONFLICT OF INTEREST}

No potential conflict of interest relevant to this article was reported.

\section{REFERENCES}

1. Stevens JC. AAEM minimonograph \#26: the electrodiagnosis of carpal tunnel syndrome. Muscle Nerve 1997;20:1477-86.

2. Werner RA, Andary M. Electrodiagnostic evaluation of carpal tunnel syndrome. Muscle Nerve 2011;44:597607.

3. Al-Shekhlee A, Shapiro BE, Preston DC. Iatrogenic complications and risks of nerve conduction studies and needle electromyography. Muscle Nerve 2003;27:517-26.

4. Rubin DI. Technical issues and potential complications of nerve conduction studies and needle electromyography. Neurol Clin 2012;30:685-710.

5. Buchberger W, Judmaier W, Birbamer G, Lener M, Schmidauer C. Carpal tunnel syndrome: diagnosis with high-resolution sonography. AJR Am J Roentgenol 1992;159:793-8.

6. Koenig RW, Pedro MT, Heinen CP, Schmidt T, Richter HP, Antoniadis G, et al. High-resolution ultrasonography in evaluating peripheral nerve entrapment and trauma. Neurosurg Focus 2009;26:E13.

7. Gellhorn AC, Carlson MJ. Inter-rater, intra-rater, and inter-machine reliability of quantitative ultrasound measurements of the patellar tendon. Ultrasound Med Biol 2013;39:791-6.

8. Reimers CD, Schlotter B, Eicke BM, Witt TN. Calf en- 
largement in neuromuscular diseases: a quantitative ultrasound study in 350 patients and review of the literature. J Neurol Sci 1996;143:46-56.

9. Schmidt R, Voit T. Ultrasound measurement of quadriceps muscle in the first year of life: normal values and application to spinal muscular atrophy. Neuropediatrics 1993;24:36-42.

10. Kamala D, Suresh S, Githa K. Real-time ultrasonography in neuromuscular problems in children. J Clin Ultrasound 1985;13:465-8.

11.Zuberi SM, Matta N, Nawaz S, Stephenson JB, McWilliam RC, Hollman A. Muscle ultrasound in the assessment of suspected neuromuscular disease in childhood. Neuromuscul Disord 1999;9:203-7.

12. Pillen S, Tak RO, Zwarts MJ, Lammens MM, Verrijp KN, Arts IM, et al. Skeletal muscle ultrasound: correlation between fibrous tissue and echo intensity. Ultrasound Med Biol 2009;35:443-6.

13. Arts IM, Pillen S, Schelhaas HJ, Overeem S, Zwarts MJ. Normal values for quantitative muscle ultrasonography in adults. Muscle Nerve 2010;41:32-41.

14. Maurits NM, Bollen AE, Windhausen A, De Jager AE, Van Der Hoeven JH. Muscle ultrasound analysis: normal values and differentiation between myopathies and neuropathies. Ultrasound Med Biol 2003;29:21525.

15. Bargfrede M, Schwennicke A, Tumani H, Reimers CD. Quantitative ultrasonography in focal neuropathies as compared to clinical and EMG findings. Eur J Ultrasound 1999;10:21-9.

16. Reimers K, Reimers CD, Wagner S, Paetzke I, Pongratz DE. Skeletal muscle sonography: a correlative study of echogenicity and morphology. J Ultrasound Med 1993;12:73-7.

17. Arts IM, van Rooij FG, Overeem S, Pillen S, Janssen HM, Schelhaas HJ, et al. Quantitative muscle ultrasonography in amyotrophic lateral sclerosis. Ultrasound Med Biol 2008;34:354-61.
18. de Krom MC, Knipschild PG, Kester AD, Thijs CT, Boekkooi PF, Spaans F. Carpal tunnel syndrome: prevalence in the general population. J Clin Epidemiol 1992;45:373-6.

19. Kim JS, Seok HY, Kim BJ. The significance of muscle echo intensity on ultrasound for focal neuropathy: the median- to ulnar-innervated muscle echo intensity ratio in carpal tunnel syndrome. Clin Neurophysiol 2016;127:880-5.

20. Scholten RR, Pillen S, Verrips A, Zwarts MJ. Quantitative ultrasonography of skeletal muscles in children: normal values. Muscle Nerve 2003;27:693-8.

21. Dumitru D, Amato AA, Zwarts M. Electrodiagnostic medicine. 2nd ed. Philadelphia: Hanley \& Belfus; 2002. p. 1062.

22. Padua L, LoMonaco M, Gregori B, Valente EM, Padua R, Tonali P. Neurophysiological classification and sensitivity in 500 carpal tunnel syndrome hands. Acta Neurol Scand 1997;96:211-7.

23. Fleiss JL. Design and analysis of clinical experiments. New York: John Wiley \& Sons; 1999. p. 1-28.

24. Schmidt WA, Schmidt H, Schicke B, Gromnica-Ihle E. Standard reference values for musculoskeletal ultrasonography. Ann Rheum Dis 2004;63:988-94.

25. Beekman R, Visser LH. Sonography in the diagnosis of carpal tunnel syndrome: a critical review of the literature. Muscle Nerve 2003;27:26-33.

26. Pillen S, Arts IM, Zwarts MJ. Muscle ultrasound in neuromuscular disorders. Muscle Nerve 2008;37:67993.

27. Simon NG, Ralph JW, Lomen-Hoerth C, Poncelet AN, Vucic S, Kiernan MC, et al. Quantitative ultrasound of denervated hand muscles. Muscle Nerve 2015;52:22130.

28. Wallwork TL, Hides JA, Stanton WR. Intrarater and interrater reliability of assessment of lumbar multifidus muscle thickness using rehabilitative ultrasound imaging. J Orthop Sports Phys Ther 2007;37:608-12. 